\title{
EFECTO DE TRES DIETAS EN EL CRECIMIENTO DE CRÍAS DE COCODRILO AMERICANO CROCODYLUS ACUTUS
}

\author{
EFFECT OF THREE DIETS ON GROWTH OF HATCHLINGS OF AMERICAN CROCODILE \\ CROCODYLUS ACUTUS
}

Hernández-Hurtado, P.S. ${ }^{1}$, Nolasco-Soria, H. ${ }^{3}$, Hernández-Hurtado, H. ${ }^{1}$, Cupul-Magaña, F.G. ${ }^{2}$, Jaime-Ceballos, B. ${ }^{4}$, Galindo-López, J. ${ }^{4}$, Carrillo-Farnés, O. ${ }^{5}$ y Vega-Villasante, F. ${ }^{2 *}$

${ }^{1}$ Reptilario Cipactli. Universidad de Guadalajara. Puerto Vallarta, Jalisco. México.

${ }^{2}$ Centro de Investigaciones Costeras. Universidad de Guadalajara. Puerto Vallarta, Jalisco. México. *fernandovega.villasante@gmail.com

${ }^{3}$ Centro de Investigaciones Biológicas del Noroeste. La Paz, B.C.S. México.

${ }^{4}$ Centro de Investigaciones Pesqueras de Cuba. La Habana. Cuba.

${ }^{5}$ Facultad de Biología. Universidad de La Habana. Cuba.

\section{PalabRas CLAVE ADICIONALES}

Alimentación reptiles. Criadero. Crecimiento. Supervivencia.

\section{RESUMEN}

Se estudió el efecto de tres dietas con sustitución de pescado marino (20 y $40 \%$ ) por hígado de res en el crecimiento y supervivencia, así como el efecto de la temperatura en el consumo de alimento de crías de Crocodylus acutus. Los cocodrilos alimentados con la dieta de $40 \%$ de hígado tuvieron el mayor crecimiento y un $100 \%$ de supervivencia, con potencial de mayor tolerancia a condiciones invernales.

\section{SUMMARY}

The effect of three diets with replacement of marine fish (20 and 40\%) for beef liver in the growth and survival, and the effect of temperature on feed intake of young Crocodylus acutus was studied. Crocodiles fed the $40 \%$ of liver had the highest growth and $100 \%$ survival, with potential for greater tolerance to winter conditions.

\section{INTRODUCCIÓN}

El estudio de la nutrición de los cocodrilos en el medio silvestre está principalmente basado en el análisis de sus contenidos estomacales y, sólo secundariamente, en la observación de su comportamiento (Huch-

Recibido: 17-1-11. Aceptado: 22-9-11.

\section{Additional KeYWORDS}

Reptile feeding. Hatchery. Growth rate. Survival rate.

zermeyer, 2003). El objetivo de este trabajo fue comparar el efecto de dos dietas, con distintas proporciones de pescado marino e hígado de res, con una dieta patrón sobre el crecimiento de crías de Crocodylus acutus en cautiverio.

\section{MATERIAL Y MÉTODOS}

La Unidad de Manejo para la Conservación de la Vida Silvestre Reptilario Cipactli, se localiza en el Centro Universitario de la Costa de la Universidad de Guadalajara ubicado entre los $20^{\circ} 42^{\prime} 19^{\prime \prime} \mathrm{Ny} 105^{\circ} 13^{\prime} 19^{\prime \prime}$ O. El estudio se llevó a cabo del 22 de octubre de 2009 al 12 de marzo de 2010 . Se utilizaron cinco acuaterrarios de $13 \mathrm{~m}^{2}$, con un espejo de agua del $40 \%$ del área total de cada uno. Las crías $(n=25)$, sembradas a la misma densidad, pertenecieron a la misma camada y eclosionaron bajo condiciones de ambiente controlado. Desde su nacimiento hasta el inicio del experimento se alimentaron con una dieta a base de pescado marino. Se tomó al filete de pescado molido (Euthy- 
nnus affinis y Caranx caballus 1:1) como el principal componente de las dietas. La mezcla de pescado marino fue la dieta control. Se efectuó su análisis proximal de acuerdo con AOAC (1990) (tabla I). Los organismos se seleccionaron al azar para conformar la población por tratamiento: a ocho $(\mathrm{n}=8) \mathrm{se}$ les suministró una dieta compuesta por $60 \%$ de pescado y $40 \%$ de hígado de res (dieta I), a ocho $(n=8)$, con una dieta con $80 \%$ de pescado y $20 \%$ de hígado de res (dieta II) y a los nueve restantes $(n=9)$ se les administró la dieta control (dieta III). Las tres dietas se presentaron para consumo cada tres o cuatro días a la misma hora (13:00-14:00 h) por un período máximo de $20 \mathrm{~h}$. El alimento suministrado fue de $25 \%$ de la biomasa total y se determinó el consumo por diferencia de peso seco. Con termómetros digitales se midió la temperatura ambiental y del agua (13:00-14:00 h). Se realizaron biometrías a los 0,60 y 127 días (longitud hocico-cola en cm y peso en g) y se calculó:

\section{Sobrevivencia, $S=(\mathrm{Nf} / \mathrm{Ni}) \times 100$}

donde:

$\mathrm{Nf}=$ número final de organismos;

$\mathrm{Ni}=$ número inicial de organismos.

Tasa de crecimiento, $\mathrm{TC}=(\mathrm{Pf}-\mathrm{Pi}) / \mathrm{Pi} \times 100$

donde:

$\mathrm{Pf}=$ peso final del organismo;

$\mathrm{Pi}=$ peso inicial del organismo.

Factor de conversión alimenticia, FCA= Alimento aparentemente consumido $(\mathrm{g}) / \mathrm{Incremento}$ en peso del organismo $(\mathrm{g})$.

Ganancia de peso por día, GPD= Pf - Pi/días

Ganancia de peso por mes, GPM $=\mathrm{Pf}-\mathrm{Pi} /$ meses

Para el análisis estadístico de la sobrevivencia, se concentraron los resultados en dos grupos: pescado (tratamiento III) y mezcla de pescado e hígado de res (tratamiento I y II). Se aplicó la prueba de diferencias de proporciones entre supervivencia de las crías en ambos grupos. Los datos de crecimiento violaron las predicciones de normalidad, por lo que se aplicó un análisis de Kruskal-Wallis y, a posteriori, una prueba de Tukey. El coeficiente de correlación de rangos de Kendall estimó la fuerza de la asociación entre el consumo con el crecimiento y la temperatura ambiental (Sokal y Rohlf, 1995). En todas las pruebas $\alpha=5 \%$.

\section{RESULTADOSYDISCUSIÓN}

La sobrevivencia de las crías para el tratamiento I fue del $100 \%(\mathrm{n}=8)$, del $62,5 \%$ para el tratamiento II $(n=5)$ y para el tratamiento III del $66,7 \%(n=6)$. Los resultados de la prueba de diferencias de proporciones para los grupos pescado (III) y mezcla de pescado e hígado de res (I y II) no permitió establecer la existencia de diferencias significativas entre la supervivencia de las crías $(\mathrm{r}=0,82)$. Los resultados demostraron que la inclusión del hígado de res resultó posi-

Tabla I. Análisis proximal de las dietas suministradas a crías de Crocodylus acutus. (Proximate analysis of diets fed to hatchlings of Crocodylus acutus).

\begin{tabular}{cccccccc}
\hline Dieta & $\begin{array}{c}\text { Humedad } \\
\%\end{array}$ & $\begin{array}{c}\text { Proteína } \\
\%\end{array}$ & $\begin{array}{c}\text { Extracto etéreo } \\
\%\end{array}$ & $\begin{array}{c}\text { Fibra bruta } \\
\%\end{array}$ & $\begin{array}{c}\text { Cenizas } \\
\%\end{array}$ & $\begin{array}{c}\text { ELN } \\
\%\end{array}$ & $\begin{array}{c}\text { Energía } \\
\text { cal/g }\end{array}$ \\
\hline I & 94,48 & 73,83 & 4,45 & 0,05 & 5,52 & 16,15 & 4734,63 \\
II & 94,18 & 77,74 & 2,31 & 0,07 & 5,84 & 14,03 & 4656,46 \\
III & 93,82 & 78,64 & 1,33 & 0,02 & 6,19 & 13,82 & 4678,14 \\
\hline
\end{tabular}

I: $60 \%$ pescado y $40 \%$ hígado de res; II: $80 \%$ pescado y $20 \%$ hígado de res; III: $100 \%$ pescado.

Archivos de zootecnia vol. 61, núm. 234, p. 314. 


\section{ALIMENTACIÓN DE CROCODYLUS ACUTUS EN CAUTIVERIO}

tiva para los organismos y difieren de los de McNease y Joanen (1981) y Bolton (1994), quienes con dietas constituidas exclusivamente por pescado han conseguido crecimientos similares o superiores. En la tabla II se observa que los tratamientos I y II propiciaron un crecimiento significativamente mayor que el tratamiento III. De manera general, el diseño de las dietas para C. acutus se basa en sustituciones parciales de pescado por otros insumos principalmente vísceras y otros componentes de mamíferos domésticos o pollo entero (Pérez y Escobedo-Galván, 2007; Pérez-Gómez et al., 2009). El alto porcentaje de inclusión de hígado de res en los tratamientos I y II, sugiere que este componente favoreció la nutrición de los organismos. El tratamiento III (pescado $100 \%$ ) provee un contenido proteico de $78,6 \%$ del total de la dieta, mientras que las grasas representan el 1,33\%. Las dietas con inclusión de hígado registran $77,7 \%$ (I) y $73,8 \%$ (II) de proteína y $2,3 \%$ y $4,4 \%$ de grasas. Con relación al contenido de carbohidratos se nota un ligero incremento cuando se incluyen porcentajes crecientes de hígado en las dietas (tabla I). Por lo anterior, se puede establecer que los contenidos proteicos son cercanos en los diferentes tratamientos. Sin embargo en relación a las grasas, el aporte de las dietas con $40 \%$ de hígado es más de tres veces superior al de la dieta III. Lo cual sugiere que este contenido de grasa es el que permite a los cocodrilillos soportar los efectos del estrés térmico, crecer y sobrevivir. Al respecto, Staton et al. (1990) encontraron que neonatos de Alligator mississippiensis criados en cautiverio, pueden beneficiarse de la presencia de grasas y carbohidratos como suplementos en su dieta. Las diferencias en el consumo total por tratamiento indican que el alimento complementado con hígado fue más consumido que el que sólo contenía pescado: I: 11,907 g; II: 10,125 g y III: 9,124 g. Asimismo, las correlaciones fueron significativas entre consumo y temperatura $(\mathrm{r}=$ $1)$, consumo y crecimiento $(\mathrm{r}=-0,54)$, así como temperatura y crecimiento $(\mathrm{r}=-0,54)$. Los valores de temperatura ambiental y del agua en los tres tratamientos fueron los mismos y oscilaron entre los 17,3 a $33,1^{\circ} \mathrm{C}$ y 19,0 a $31,5^{\circ} \mathrm{C}$, respectivamente. Por su parte, el consumo máximo de alimento se registró entre los meses de octubre a diciembre de 2009 y febrero a marzo de 2010 y, el mínimo, entre diciembre 2009 a febrero de 2010. Cuando se relacionan los datos obtenidos en crecimiento con los de temperatura ambiental y del agua, se observa que la disminución de la temperatura ambiental afectó el consumo en todos los tratamientos. Se sugiere entonces que la temperatura ambiental a la que se desarrollaron los reptiles en cada uno de los experimentos, fue un factor determinante para observar diferencias entre

Tabla II. Crecimiento de crías de Crocodylus acutus alimentados con dietas a base de pescado e hígado de res. (Growth of hatchlings of Crocodylus acutus fed with diets based on marine fish and beef liver).

\begin{tabular}{ccccccc}
\hline & \multicolumn{2}{c}{ Peso $(\mathrm{g})^{*}$} & \multicolumn{2}{c}{ Tasa de } & \multicolumn{3}{c}{ incremento peso (g) } & \multirow{2}{*}{ FCA } \\
Dieta & inicial & final & crecimiento $\%$ & día & mes & \\
\hline I & $133,75 \pm 5.8^{\mathrm{a}}$ & $344,5 \pm 92,2^{\mathrm{a}}$ & 157 & 1,65 & 52 & 6,9 \\
II & $156,25 \pm 11.6^{\mathrm{a}}$ & $335,75 \pm 80,7^{\mathrm{b}}$ & 114 & 1,41 & 44,8 & 7,0 \\
III & $155,0 \pm 29.4^{\mathrm{a}}$ & $244,0 \pm 83,4^{\mathrm{c}}$ & 57 & 0,70 & 22,2 & 9,4 \\
\hline
\end{tabular}

*Media \pm desviación estándar.

${ }^{a b c}$ En columnas, letras distintas indican diferencias significativas $(p<0,05)$.

FCA: Factor de conversión alimenticia. 
este estudio y los citados anteriormente; debido a que los cocodrilos en cautiverio crecen y asimilan mejor los nutrientes cuando su temperatura corporal (reflejo de la ambiental) se encuentra alrededor de los 32 a $33^{\circ} \mathrm{C}$ (Huchzermeyer, 2003). La temperatura mínima observada en este estudio para los tres estanques fue de $18^{\circ} \mathrm{C}$ en promedio, resultado de practicar el ensayo durante los meses fríos de octubre y marzo. Al respecto, Kanui et al. (1991) registraron la disminución y cese del crecimiento en $C$. niloticus a $25^{\circ} \mathrm{C}$, y observaron también la ralentización del tránsito gastrointestinal a esta misma temperatura. Para C. acutus se ha observa-

\section{BIBLIOGRAFÍA}

AOAC. 1990. Official methods of analysis. Association of Official Analytical Chemists. Washington, DC. $1230 \mathrm{pp}$.

Bolton, M. 1994. La explotación del cocodrilo en cautividad. Guía FAO Conservación № 22. Italia. $156 \mathrm{pp}$.

Huchzermeyer, F.W. 2003. Crocodiles: biology, husbandry and diseases. CABI Publishing. Londres. 337 pp.

Kanui, T., Mwendia, C., Aulie, A. and Wanyoike, M. 1991. Effects of temperature on growth, food uptake and retention time of juvenile Nile crocodiles (Crocodylus niloticus). Comp Biochem Phys, 99A: 453-456.

McNease, L. and Joanen, T. 1981. Nutrition of alligators. Proc Alligator Production Conf Gainsville, Florida, 1: 15-28.

Pérez, O. y Escobedo-Galván, A. 2007. Creci- do que durante la temporada invernal el tránsito gastrointestinal disminuye de forma marcada, llegando incluso a detenerse, provocando timpanismo y la muerte en cuestión de días.

\section{AGRADECIMIENTOS}

Al Proyecto COECYTJAL-UdeG 062009-661, beca de doctorado de CONACYT 194981, Sociedad Cooperativa de Pescadores del Rosita y Rastro municipal de Puerto Vallarta. A la MSc. Olimpia Chong Carrillo la preparación del manuscrito. Dedicado a la memoria del M.C. José Galindo López.

miento en cautiverio de Crocodylus acutus (Cuvier, 1807) en Tumbes, Perú. Rev Peruana Biol, 14: 221-223.

Pérez-Gómez, M., Naranjo-López, C., Reyes-Tur, B. y Vega-Ramírez, I. 2009. Influencia de dos tipos de dietas sobre la talla y el peso corporal en neonatos de Crocodylus acutus Cuvier, 1807 (Crocodylidae: Crocodylia) del zoocriadero de Manzanillo, Cuba. Acta Zool Mex (n.s.), 25: 151-160. Sokal, R.R. and Rohlf, F.J. 1995. Biometry: the principles and practice of statistics in biological research. $3^{\text {rd }}$ edition. W.H. Freeman and Co. New York. 887 pp.

Staton, M.A., Edwards, H.M., Brisbin-Junior, I.L., Joanen, T. and Mcnease, L. 1990. Protein and energy relationships in the diet of the American alligator (Alligator mississippiensis). J Nutr, 120: $775-85$. 